\title{
Evaluation of a 5-year multidisciplinary approach for the treat- ment of childhood obesity
}

\author{
Véronique Beauloye
}

Professor - «Chef de Clinique Associe» in the Division of Paediatric Endocrinology at the Catholic University of Louvain

Introduction: Childhood obesity prevalence is increasing almost universally with little evidence to support any specific treatment. Information on long-term outcome of obesity treatment in children and adolescents is needed.

Objective: To examine the efficacy of our multidisciplinary approach for treating obesity in childhood.

Method: In 2000, we set up a multidisciplinary programme for overweight children and adolescents consisting in join consultations, where each young and his family (all adults taking care of the child) were seen by both a psychologist and a paediatric endocrinologist. Then, the young and his family went to the dietitian. Before each session, we summarized the situation of each patient. A letter including our common decision was sent to the patient and his family within the month following the consultation. Our approach is an individually adapted (specific for each patient) familytargeted behavioural lifestyle interventions program. We emphasized on the importance of physical exercise in daily life. Physical activity is also enhanced through inscription in sport clubs or physiotherapy. No special diets were prescribed. Psychosocial determinants such as self-esteem, ethnic and economic factors, family's capacity to change behaviour were also taken into account in the global management of the patients. In the present study, we reviewed all the patients followed between 2002 and 2007, whom have had almost two multidisciplinary consultations and at least a one-year follow-up.

doi:10.1017/S136898001200170X

\section{Residential management}

\section{Ann Tanghe}

Zeepreventorium VZW, De Haan, Belgium

Most children confronted to obesity remain overweight when they enter adulthood. Evidence today demonstrates that continued obesity leads to serious health problems as adult obesity is known to be very resistant to treatment. Hence, since 1994 the Zeepreventorium in De Haan attempts to offer a multidisciplinary treatment and coaching in residential care to severely obese children.
Results: In all, 144 children (59 boys, 85 girls; mean age $10 \cdot 5$ (SD $3 \cdot 1$ ) years; mean BMI-SDS $2 \cdot 73$ (SD 0.63)) out of the 428 children seen during 2002-2007 met the inclusion criteria. At the second consultation (approximately after 3-6 months), BMI-SDS was decreased in 53\% of the patients, remained unchanged in $31 \%$ and was increased in $16 \%$. Mean follow-up was $2 \cdot 2$ years (range $1 \cdot 0-6 \cdot 7$ years) with an average of $3 \pm 1$ visits per year. Assiduity to follow-up did not depend of the weight change at the second consultation $(P=0 \cdot 33$, NS). We showed a decrease in BMI-SDS by $3 \cdot 3 \%$ after 3 months, $5 \cdot 0 \%$ after 6 months, $6 \cdot 7 \%$ after $9-24$ months and $7 \cdot 7 \%$ after 27-42 months of follow-up with a mean BMI-SDS (2.51 (SD 0.06)) at the end of follow-up. At the latest consultation, BMI-SDS was decreased, unchanged or increased, respectively, in $67 \%, 10 \%$ and $23 \%$ of the patients. Initial BMI-SDS, age at the first evaluation, sex and number of consultations per year did not influence our results $(P=0 \cdot 31, P=0 \cdot 42, P=0 \cdot 36, P=0 \cdot 89$, respectively). Weight loss was mainly observed during the first 6 months of treatment and was sustained at long term. The weight changes observed between the first and second consultation were predictive of the results at the end of follow-up.

Conclusions: We showed that our family-based combined approach provide a short- and long-term decrease in overweight in both children and adolescents.
Although inpatient treatment is a drastic intervention for obese children and youngsters, a lot of them are interested in following this long-term treatment programme, which they (and their parents) often consider as their last remaining option.

Admission criteria: Severely obese children and adolescents from 5 up to 18 years are admitted to this 
residential treatment. 'Severe' meaning a BMI >97th percentile for age and gender for youngsters under 16 years of age and a BMI above 35 or above 30 with physical comorbidities for adolescents above 16 years.

All patients have already been enrolled in an outpatient treatment which failed or which they abandoned.

Involvement of at least one supportive parent is needed to provide continued support during the time periods the child spends at home. For some adolescents exceptions are made when their own adherence to the programme is considered high enough. The younger the patient, the more the parents will have to get involved.

Special attention is requested for mentally retarded children. Admission is only possible for children with an IQ over 70. For all children not fitting into the program in case their IQ is inferior to 70 , suggestions are made by the Zeepreventorium De Haan for the special schools in which they often stay. To determine whether or not children and their supportive family are motivated enough, the rule of thumb is applied that children have to decide themselves whether or not they want (are in favour of) an inpatient treatment. Therefore, it is important to give sufficient time for reflection to the children and their parents, prior to their admission.

Treatment ingredients: Treatment goal is inducing a healthy lifestyle. Within a medical framework patients and their supporting family are invited to put their lives in 'balance': this means getting the energy balance right by introducing daily physical activity and changing dietary habits on a strong psychoeducational basis with strong cognitive behavioural components. During the lecture the four elements of treatment will be discussed. All members of staff are specialists who learned to cope with their own attitudes and preconceptions towards obese children and their families.

Treatment results: Studies are being performed with (amongst others) staff members of Prof. Dr C Braet, Ghent University, Department of Developmental, Personality and Social Psychology and Dr B Deforche, Department of Movement and Sports Sciences. Longterm outcome studies as well as studies to determine predictors of weight loss and patient characteristics will be discussed.

Remaining challenges: Despite a decade of experience in the treatment of children and youngsters with severe obesity, the Zeepreventorium De Haan still has to face a lot of challenges! The evidence for psychopathology and the question of how to implement treatment for these comorbidities, the work with the (often obese) parents of obese youngsters, the choice for bariatric surgery in the case of obese adolescents, the modest weight results in the long term requesting greater selfacceptance, finding a concordance between living with this chronic disease and the enormous demands of our society. All these are important issues to be considered and addressed.

\section{Ten years research in the care-system for children and youngsters with eating/weight problems in Flanders: a vision for long-term management}

\section{Ann Vandeputte}

Initiator and coordinator of the expertise centrum 'eetexpert.be vzw'

About 10 years ago, the Flemish Department for Welfare initiated a series of projects to explore and improve the health care for children and youngsters with eating disorders and weight problems.

To explore existing care pads, we have contacted a wide range of professionals: teams of school health centres (physicians, nurses and psychologists), general practitioners, primary care workers, paediatricians, dietitians, psychologists, community mental health teams and residential or inpatient settings. This survey revealed a good system for early detection, but also the lack of knowledge about weight-related problems in primary care, a feeling of incompetence to manage these problems in secondary care, a need for better overview of referral possibilities and the concentration of specialized care around some cities. International recommendations emphasize multidisciplinarily, continuity and stepped care for this group of patients with eating/weight problems:

- Because of the physical, psychosocial and dietary aspects, good multidisciplinary teamwork is wanted.

- Because of their often early development and persistence over many years, these problems necessitate a continuity of care over several life stages. 